\title{
Individual differences in rhesus monkeys' demand for drugs of abuse
}

\author{
Mikhail N. Koffarnus', Amy Hall ${ }^{2} \&$ Gail Winger ${ }^{2}$ \\ Behavioral Pharmacology Research Unit, Johns Hopkins University School of Medicine, Baltimore, MD, USA' and Department of Pharmacology, University of \\ Michigan, Ann Arbor, MI, USA²
}

\begin{abstract}
A relatively small percentage of humans who are exposed to drugs of abuse eventually become addicted to or dependent on those drugs. These individual differences in likelihood of developing drug addiction may reflect behavioral, neurobiological or genetic correlates of drug addiction and are therefore important to model. Behavioral economic measures of demand establish functions whose overall elasticity (rate of decrease in consumption as price increases) reflects the reinforcing effectiveness of various stimuli, including drugs. Using these demand functions, we determined the reinforcing effectiveness of five drugs of abuse (cocaine, remifentanil, ketamine, methohexital and ethanol) in 10 rhesus monkeys with histories of intravenous drug-taking. There was a continuum of reinforcing effectiveness across the five drugs, with cocaine and remifentanil showing the most reinforcing effectiveness. There was also a continuum of sensitivity of the monkeys; two of the 10 animals, in particular, showed greater demand for the drugs than did the remaining eight monkeys. In addition, monkeys that demonstrated greater demand for one drug tended to show greater demand for all drugs but did not show a similar relatively greater demand for sucrose pellets. These findings suggest that the tendency to find drugs to be reinforcing is a general one, not restricted to particular drugs and also, that a minority of animals show a substantially enhanced sensitivity to the reinforcing effects of drugs. The possibility that differences in responsiveness to the reinforcing effects of drugs may form the basis of individual differences in drugtaking in humans should be considered.
\end{abstract}

Keywords Behavioral economics, drug addiction, drug self-administration, individual differences, reinforcing effectiveness, rhesus monkeys.

Correspondence to: Mikhail Koffarnus, Behavioral Pharmacology Research Unit, Johns Hopkins University School of Medicine, 5200 Eastern Ave., Suite 142W, Baltimore, MD 21224-6823, USA. E-mail: mkoffar1@jhmi.edu

\section{INTRODUCTION}

One important aspect of drug addiction in humans is that it occurs in a minority of individuals who engage in drugtaking behavior. Anthony, Warner \& Kessler (1994) surveyed Americans between the ages of 15 and 54 and reported that $75.6 \%$ of this population had tried tobacco, and of these participants, $31.9 \%$ could be considered dependent. A larger number, $91.5 \%$ of the population, had tried alcohol, and 15.4\% developed dependence. Fewer individuals had tried cocaine $(16.2 \%)$ or heroin (1.5\%), but a substantial percentage of users $(16.7 \%$ and $23.1 \%$, respectively) became dependent on these drugs. Given that not all humans who try drugs become addicted or dependent, it is worthwhile to attempt to identify individual differences in drug taking by animals that might reflect those differences in humans.
Research on individual differences in behavior, and how these differences might relate to addiction proclivities, is currently gaining increasing attention, typically using rats as subjects and stimulants as the drugs of abuse. Some of the behaviors on which rats differed were the degree of locomotion they engaged in when exposed to a novel environment (Piazza et al. 1989, 1990), whether they responded more to a stimulus that was paired with a reinforcer (sign tracking) or to the place where the reinforcer was delivered (goal tracking) (Flagel et al. 2008) and how impulsive they were in specific reaction time tasks (Belin et al. 2008). Each of these behaviors was reflected in addiction-related outcomes. For example, rats that showed more novelty-induced locomotion acquired the ability to self-administer amphetamine, whereas low responding rats did not (Piazza et al. 1989, 1990). Rats that sign-tracked 
showed somewhat more behavioral sensitization to cocaine over seven days of drug administration than did the goal-tracking rats (Flagel et al. 2008).

The reinforcing effects of drugs are fundamental to the development of drug addiction and intrinsic to most rodent models of addiction-like behavior. Nevertheless, individual differences in responsiveness to drugs' reinforcing effects are rarely investigated as potential contributors to addiction. Studies of genetic bases of addiction notwithstanding (e.g. Uhl 2006; Osby et al. 2010), this question is difficult to approach in the absence of a quantitative measure of the relative reinforcing effectiveness of drugs (see Stephens et al. 2010 for review). Behavioral economic procedures permit quantification of reinforcing effects, placing a relative value on different drug reinforcers or on individual differences in the reinforcing effect of the same drug. This is done with demand curves, which plot the amount of reinforcer consumed as a function of the price (response requirement and dose) of the drug. As long as doses are used that serve as reinforcers, these curves are independent of the magnitude (dose) of the reinforcer, allowing each reinforcing stimulus to be given a single number that reflects its reinforcing effectiveness: either the change in elasticity (slope) of the demand curve as price increases or the point along the increasing price axis where the most responses are made $\left(P_{\max }\right)$ (Hursh \& Silberberg 2008). Relatively inelastic demand curves and higher $P_{\max }$ values indicate a reinforcer with more effectiveness than that indicated by more elastic demand curves.

Demand curve analysis has the additional advantage of permitting concentrated evaluation of relatively few numbers of subjects as is often required in nonhuman primate research. The use of demand curve analysis to discern individual differences in the reinforcing effects of drugs in these animals is potentially instructive in attempts to identify idiosyncratic drugorganism interactions that might eventually be applied to human drug abuse issues. Among the questions in the current study were whether the animals showed individual differences in responsiveness to the reinforcing effects of drugs, whether monkeys that showed greater demand for one drug tended to show greater demand for other drugs or whether some monkeys have high demand for only one drug and whether the tendency to show high demand for a drug carried over to demand for non-drug stimuli such as sucrose pellets or saline injections. Comparisons were also made among animals with respect to the amount of drug each self-administered under the lowest price condition in order to ascertain whether this measure provided similar answers to these questions.

\section{MATERIALS AND METHODS}

\section{Subjects}

Subjects were 10 adult rhesus monkeys (Macaca mulatta), eight males and two females (BU and HI). The monkeys were fed high-protein monkey biscuits (LabDiet FiberPlus, Brentwood, MO, USA) twice daily and fresh fruit or vegetables once daily. Individual healthy adult body weights, as determined by veterinary staff, were maintained and the monkeys were not systematically fooddeprived. Water was continuously available and novel environmental enrichment was provided weekly. All monkeys had a history of drug self-administration, and the duration and extent of their histories is indicated in Table 1.

The University of Michigan is accredited by the American Association for the Accreditation of Laboratory Animal Care; procedures used in this experiment were conducted in accordance with the National Research Council's (1996) Guide for the Care and Use of Laboratory Animals and approved by the University Committee on Care and Use of Animals.

\section{Apparatus}

The monkeys were individually housed and tested in stainless steel cages that measured $83.3 \mathrm{~cm} \times 76.2 \mathrm{~cm} \times$ $91.4 \mathrm{~cm}$. Each cage was equipped with a response panel located on the left wall, approximately $10 \mathrm{~cm}$ from the front and $19 \mathrm{~cm}$ from the bottom of the cage. The panel contained three horizontally aligned levers that could be activated by approximately $0.10-0.15 \mathrm{~N}$ of force. Three stimulus lights, each of which was centered above a lever, were $2.5 \mathrm{~cm}$ in diameter and spaced $1.5 \mathrm{~cm}$ apart. Each light consisted of a circular opening that was covered with translucent plastic and capable of being illuminated from behind by $5-W$ colored bulbs. During the present study, only one lever was active, and the light over that lever and the center light were used.

Monkeys were implanted with indwelling intravenous catheters (Murdock Industrial Inc, Akron, OH, USA) that passed subcutaneously from the implanted veins to exit sites on the monkeys' backs. Each catheter was fed through a stainless steel, flexible tether that was attached to the back of the Teflon mesh jacket (Lomir, Notre-Damede-l'Île Perrot, Quebec, Canada) worn by each monkey. The other end of the tether was attached to the back wall of the cage. Once the catheter exited the tether behind the cage, it connected to a pump that was capable of infusing the currently available drug into the catheter. The infusion pump (Watson-Marlow model Sci-Q 400, Wilmingon, MA, USA) delivered $0.2 \mathrm{ml}$ of solution per second. Each delivery lasted five seconds (totaling a 1-ml injection volume). Control of experimental sessions was provided 


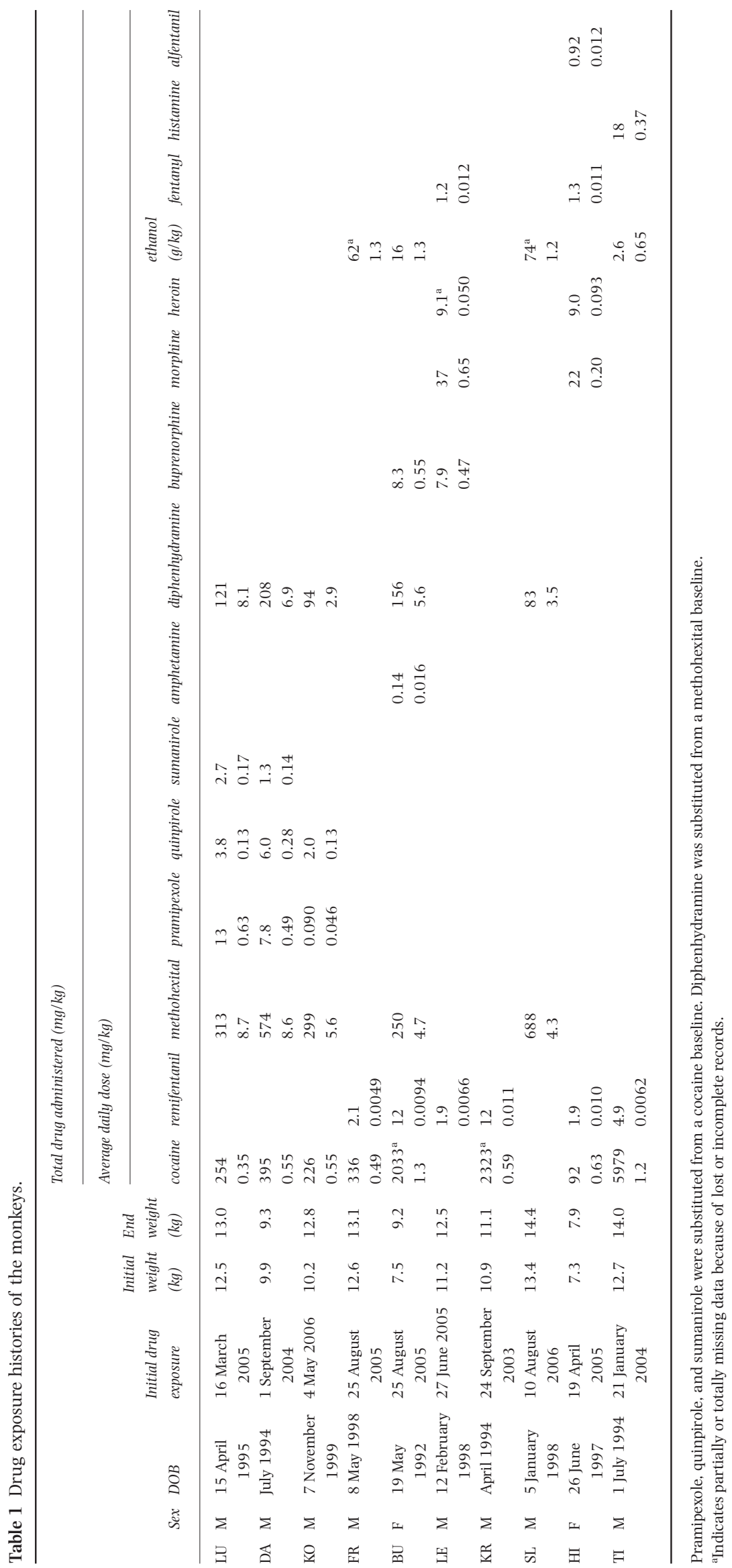


by Med-PC (Med-Associates, Georgia, VT, USA), interfacing and software installed on computers located in an adjacent room.

Once all drugs and saline had been studied, demand for $300 \mathrm{mg}$ sucrose pellets was evaluated. The stimulus lights and response levers were the same during testing with sucrose pellets as they had been during testing of the various drugs. A $43-\mathrm{cm}$ square panel was added to the housing cage, above and to the right of the stimulus light and lever panel. This panel contained a food dispenser (Model G5210, Gerbrands Corporation, Arlington, MA, USA) on the outside and a pellet receptacle on the monkeys' side. Although nine of the 10 monkeys responded readily for sucrose pellets without requiring food deprivation, sucrose-maintained responding was not stable in one monkey (SL) unless he was food-restricted from 40 chows per day to 10 chows per day. He did not lose weight during the one-month period his chow was restricted.

\section{Experimental procedures}

The monkeys accessed the test drugs during two, 120minute sessions each day, which were approximately four hours apart. Ethanol was an exception in that because it has a fairly long duration of action; it was available during only one 120-minute session each day. The beginning of each session was indicated by the onset of the rightmost, red stimulus light. In the presence of this light, a predetermined number of responses on the right lever caused the red light to be extinguished and a drug infusion to be initiated. During the five-second drug infusion, a green, center stimulus light was illuminated. Ethanol was again an exception, with the concentration remaining constant at $15 \% \mathrm{w} / \mathrm{v}$, and the infusion duration varying depending on the weight of the monkey. Following the infusion, all stimulus lights were turned off for 10 seconds, during which time responses had no programmed consequence. The red light was then illuminated again, and responses were again effective in producing a drug infusion.

The monkeys were required to make a fixed number of responses to earn each drug injection in daily sessions. The requirements were 10, 32, 100, 320, 562 and 1000 responses per injection. The ratio value remained constant throughout a session, and each ratio value was in effect for two consecutive sessions; the ratio sequence across sessions was always ascending. Once a ratio of 1000 had been presented, the ratio value was reset to 10 and the series was repeated with another drug, saline or finally, sucrose pellets.

\section{Drugs}

Five drugs (cocaine, remifentanil, methohexital, ketamine and ethanol) and saline were made available to the monkeys. Cocaine was provided by the National Institute on Drug Abuse (Bethesda, MD). Remifentanil was purchased from the University of Michigan Hospital Pharmacy. Methohexital and ketamine were purchased from Henry Schein Animal Health (Melville, NY, USA, http:// www.henryschein.com). Ethanol was purchased from Fisher Scientific (Rockford, IL, USA, http://www. fishersci.com). The order of reinforcer presentation to the different animals was non-systematic and is shown in Table 2. All drugs were dissolved in sterile, normal saline. All drugs except ethanol were delivered in a volume of $1 \mathrm{ml}$ over five seconds in concentrations that varied depending on the animals' weights and the dose. Ethanol was prepared as a $15 \% \mathrm{w} / \mathrm{v}$ solution; so for the $0.05 \mathrm{gm} /$ $\mathrm{kg} /$ injection dose used, the infusion volume of ethanol was $1 \mathrm{ml}$ per $3 \mathrm{~kg}$ of body weight $(2.37$ to $4.8 \mathrm{ml} /$ injection; 11.8 to 24 seconds infusion duration).

Table 2 The order that the five drugs and saline were presented to each monkey.

\begin{tabular}{|c|c|c|c|c|c|c|}
\hline & \multicolumn{6}{|c|}{ Drug presentation order } \\
\hline & 1 & 2 & 3 & 4 & 5 & 6 \\
\hline $\mathrm{LU}$ & Remifentanil & Saline & Methohexital & Ketamine & Ethanol & Cocaine \\
\hline DA & Methohexital & Saline & Ketamine & Remifentanil & Cocaine & Ethanol \\
\hline KO & Methohexital & Ketamine & Saline & Cocaine & Remifentanil & Ethanol \\
\hline FR & Remifentanil & Methohexital & Ethanol & Ketamine & Saline & Cocaine \\
\hline $\mathrm{LE}$ & Methohexital & Saline & Remifentanil & Ketamine & Ethanol & Cocaine \\
\hline KR & Saline & Ketamine & Methohexital & Ethanol & Remifentanil & Cocaine \\
\hline $\mathrm{BU}$ & Methohexital & Ketamine & Ethanol & Remifentanil & Saline & Cocaine \\
\hline SL & Methohexital & Ketamine & Cocaine & Remifentanil & Saline & Ethanol \\
\hline $\mathrm{TI}$ & Ketamine & Cocaine & Methohexital & Remifentanil & Ethanol & Saline \\
\hline $\mathrm{HI}$ & Methohexital & Cocaine & Saline & Remifentanil & Ethanol & Ketamine \\
\hline
\end{tabular}


Because elasticity of demand functions is not modified by dose of the commodity offered (Hursh \& Winger 1995), single doses of each drug and a single sucrose pellet size were evaluated. The doses (solution concentrations in parentheses) were remifentanil $0.0001 \mathrm{mg} / \mathrm{kg} /$ injection $(0.00071$ to $0.00144 \mathrm{mg} / \mathrm{ml})$, ketamine $0.03 \mathrm{mg} / \mathrm{kg} /$ injection $(0.213$ to $0.432 \mathrm{mg} / \mathrm{ml})$, methohexital $0.1 \mathrm{mg} / \mathrm{kg} /$ injection (0.71 to $1.44 \mathrm{mg} / \mathrm{ml}$ ) and ethanol $0.05 \mathrm{gm} / \mathrm{kg} /$ injection (always $15 \% \mathrm{w} / \mathrm{v}$ ). Cocaine was available at a dose of $0.01 \mathrm{mg} / \mathrm{kg} /$ injection ( 0.071 to $0.144 \mathrm{mg} / \mathrm{ml}$ ) for all the monkeys except BU and LE who, because they did not respond reliably at this dose at larger ratio values, were evaluated at a dose of $0.03 \mathrm{mg} / \mathrm{kg} /$ injection (0.225 to $0.375 \mathrm{mg} / \mathrm{ml}$ ).

\section{Data analysis}

The dependent variable of interest was the consumption of each drug or the number of ratios completed during the two-hour sessions of drug availability. Data obtained from the two consecutive sessions at each fixed ratio (FR) were averaged. These consumption data are expressed in the form of demand curves throughout this article.

Normalized demand curves (Hursh \& Winger 1995; Hursh \& Silberberg 2008) in which the point of origin on the log-log curve was set equal to 100 were used to compare elasticity of demand for the several drugs examined in this study. Consumption data were normalized to consumption at an FR 10 with the equation

$$
Q=100 * \frac{Y_{n}}{Y_{10}}
$$

where normalized consumption $(Q)$ was equal to consumption at FR $n\left(Y_{n}\right)$ divided by consumption at FR 10 $\left(Y_{10}\right)$ expressed as percent. Price was normalized with the equation

$$
P=\frac{\mathrm{FR} * Y_{10}}{100}
$$

where $P$ is normalized price, FR is the FR value, and $Y_{10}$ is consumption at an FR 10. These normalized data were then fit to the equation

$$
\log Q=\log Q_{0}+k\left(e^{-a P}-1\right)
$$

where $Q$ is reinforcer consumption at $P$ price, $Q_{0}$ is the level of consumption as $P$ approaches 0 , and $k$ and $a$ are fit parameters. Since data were normalized to a consumption of $100, Q_{0}$ was set to 100 for all analyses. The $a$ parameter indicates the elasticity of the curve, or the rate that consumption declines with increases in price, and the $k$ parameter represents the span of the function in $\log _{10}$ units. A common span of $k=2.363$ was used for all analyses, which was experimentally derived by obtaining the best fit curves to the mean data for saline, sucros pellets and each drug in this article using GraphPad Prism 5 (La Jolla, CA, USA). In this analysis, the $a$ parameter was allowed to vary and a single shared $k$-value was fit to all data sets. This left a single free parameter $(a)$ that quantified elasticity of demand, the proposed measure of reinforcer effectiveness. The price that supported the most overall responding was also computed. This value, $P_{\max }$, was determined for each function by taking the first derivative of equation 3 and evaluating at $\mathrm{Y}=-1$ with $a$ equal to the fitted value for the curve of interest.

To compare correlations between two parameters that were both subject to experimental variability, Pearson product-moment correlations and Deming regressions were conducted using GraphPad Prism 5. The distribution of $P_{\max }$ values was not normal in the current experiment so the values were log-transformed when used as the basis of statistical comparisons. Standard scores for $\log P_{\max }$ values and number of reinforcers earned at FR 10 were calculated by determining the number of standard deviations each subject's value was from the mean value of all subjects. This was done individually for each drug and for saline and sucrose pellets.

\section{RESULTS}

As we have shown previously (Hursh \& Winger 1995), $P_{\max }$ was largest (indicating relatively greater reinforcing effectiveness) for cocaine and remifentanil and did not differ between these two drugs $\left(95 \%\right.$ CI around $P_{\max }$ overlapped; see bottom panel of Fig. 1). The $P_{\max }$ values for sucrose pellets, methohexital and ketamine were approxi-

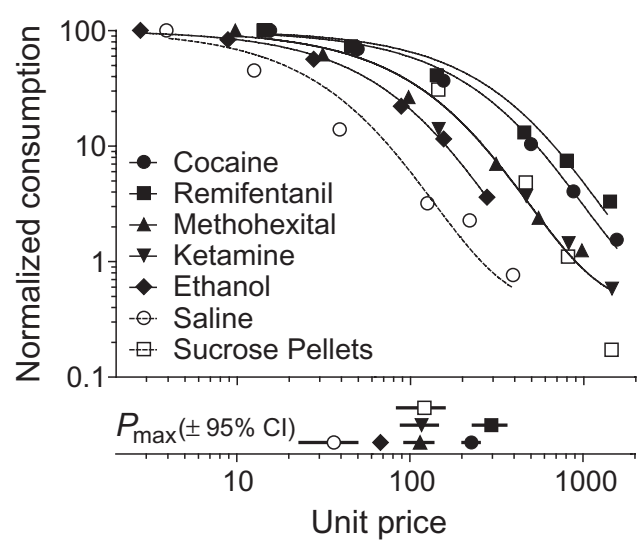

Figure I Normalized demand for cocaine (-), remifentanil $(\mathbf{\square})$, methohexital $(\boldsymbol{\Delta})$, ketamine $(\boldsymbol{\nabla})$, ethanol $(\boldsymbol{\nabla})$, saline $(\bigcirc)$ and sucrose pellets $(\square)$. Non-linear regression lines using equation 3 are shown (Qo was set to 100, k was a shared best-fit value common to the entire data set and was equal to 2.363 and a was allowed to vary for each reinforcer). Note that the regression lines for methohexital, ketamine and sucrose pellets lie nearly on top of one another and are difficult to distinguish. The $P_{\max }( \pm 95 \% \mathrm{Cl})$ values derived from each curve are displayed in the bottom panel 
Table $3 P_{\max }$ values for each subject.

\begin{tabular}{lllllrrr}
\hline & Cocaine & Remifentanil & Methohexital & Ketamine & Ethanol & Saline & Sucrose \\
\hline LU & $468.56^{* * *}$ & $372.08^{* * *}$ & $201.22^{* *}$ & $173.88^{* *}$ & $187.46^{*}$ & 95.24 & $46.25^{*}$ \\
DA & $308.26^{* * *}$ & $544.15^{* * *}$ & $251.88^{* * *}$ & $186.70^{* * *}$ & $89.68^{*}$ & 50.64 & 81.32 \\
KO & $235.82^{* * *}$ & $267.87^{* * *}$ & $134.81^{* * *}$ & $138.37^{* * *}$ & $55.83^{* * *}$ & 15.55 & $182.85^{* * *}$ \\
FR & $201.22^{* * *}$ & $272.33^{* * *}$ & $118.45^{* * *}$ & $148.93^{* * *}$ & $59.39^{* * *}$ & 7.97 & $90.10^{* *}$ \\
LE & $100.13^{* *}$ & $143.28^{* * *}$ & $83.75^{* * *}$ & $143.10^{* * *}$ & $50.37^{* * *}$ & 11.33 & $131.73^{* * *}$ \\
KR & $147.03^{* * *}$ & $307.81^{* * *}$ & 38.56 & 32.68 & $42.60^{* *}$ & 15.94 & $116.95^{* * *}$ \\
BU & $194.26^{* * *}$ & $217.76^{* * *}$ & $76.26^{*}$ & $101.05^{* *}$ & See text & 40.45 & $112.28^{* *}$ \\
SL & $172.84^{* *}$ & $180.84^{* *}$ & 32.65 & 44.86 & 32.07 & 19.38 & 91.43 \\
TI & 114.11 & $261.54^{*}$ & 19.57 & 26.59 & 31.34 & 24.36 & $271.34^{*}$ \\
HI & $98.33^{* * *}$ & $122.03^{* * *}$ & $52.51^{*}$ & $61.62^{* *}$ & $46.78^{*}$ & 16.50 & $37.30^{*}$ \\
\hline
\end{tabular}

Asterisks indicate significantly different demand elasticity compared with saline as determined by non-linear regression $\left({ }^{*} P<0.05\right.$, ${ }^{* *} P<0.01$, $* * * P<0.001)$.

mately the same and each was less than those for remifentanil and cocaine. Ethanol maintained a $P_{\max }$ that was larger than that maintained by saline but smaller than that maintained by all other drugs and sucrose pellets.

When evaluated for individual monkeys, only remifentanil maintained a larger $P_{\max }$ than did saline for all 10 monkeys. As shown in Table 3, nine of the 10 monkeys showed a larger $P_{\max }$ for cocaine than saline and seven of 10 showed a larger $P_{\max }$ for methohexital, ketamine, ethanol and sucrose pellets than for saline. One monkey, LU, showed a significantly smaller $P_{\max }$ (relatively lower reinforcing effectiveness) for sucrose pellets than for saline, and one monkey, BU, did not selfadminister any ethanol at any FR greater than FR 10, preventing the calculation of demand parameters.

A continuum was evidenced in the demand of the monkeys for the drugs (Fig. 2a). This was a general rather than drug-selective effect, with the monkeys that showed relatively greater reinforcing effectiveness of any one drug also demonstrating relatively greater reinforcing effectiveness for the other four drugs. The monkeys LU and DA showed the largest $P_{\max }$ for each of the drugs relative to the other monkeys, whereas monkeys HI, TI and SL had $P_{\max }$ values that were consistently smaller than average. Although the monkeys arranged themselves in a somewhat similar order when demand for saline was calculated, this was not the case when demand for sucrose pellets was determined. The standard deviation scores for sucrose pellets did not conform to those shown for the drugs.

The tendency for demand elasticity and therefore $P_{\max }$ to correlate across drugs of varying classes but not sucrose pellets is shown in Fig. 3 and Table 4. When the $P_{\max }$ values obtained with individual subjects for each drug were compared with the mean $P_{\max }$ value of the other drugs, positive correlations were the result in each case. These correlations were statistically significant in
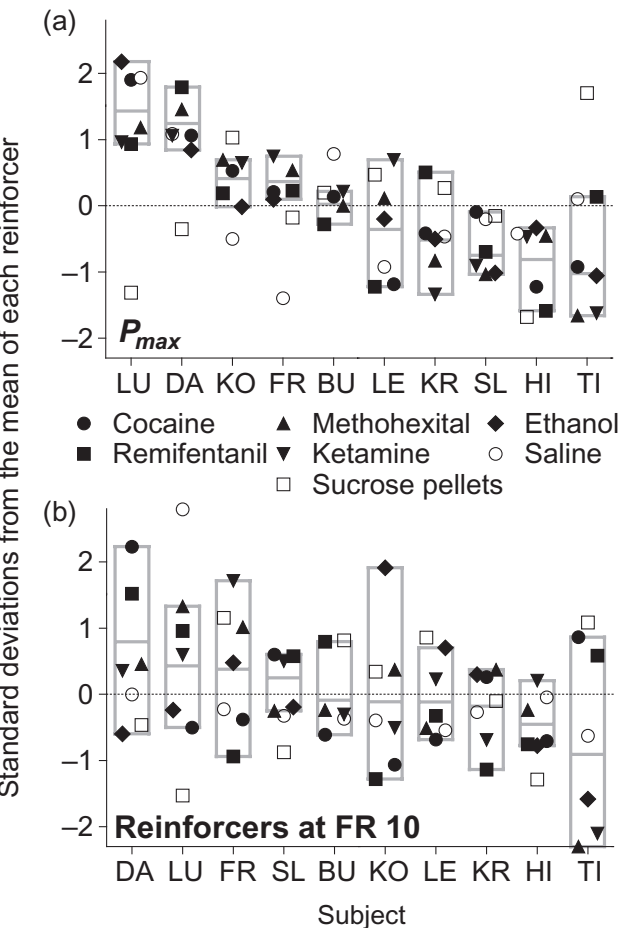

Figure 2 The position of each subjects' log $P_{\max }$ values (a) and reinforcers earned at an FR 10 (b) relative to the group mean of values for each reinforcer. Number of standard deviations from the mean is plotted on the ordinate for each measure, and points represent each drug for each subject. The gray boxes surround the range of values for the drugs only (filled symbols), and the gray bars represent the mean of the drugs only. The open circles (saline) and open squares (sucrose pellets) are displayed for comparison purposes

the case of cocaine, methohexital, ketamine and ethanol (all $P<0.01$ ). This correlation was positive for remifentanil ( $r=0.536)$ but not statistically significant with this sample size. This underscores the finding that demand for drugs appeared to be a trait-like phenomenon; the monkeys that showed relatively greater reinforcing effec- 
Figure 3 Correlations between the $P_{\max }$ value obtained for each drug and the mean $P_{\max }$ value for the other drugs (a, b, d, e, g). For example, the abscissa in panel a represents the $P_{\max }$ value for cocaine for each subject, and the ordinate represents the mean $P_{\max }$ value of remifentanil, methohexital, ketamine and ethanol. The correlations between the $P_{\max }$ value for sucrose pellets (c) and saline (f) and the mean $P_{\max }$ value for all five drugs are also shown. Deming regression lines and the Pearson $r$ for each correlation are displayed on the panel along with the statistical significance of the correlation coefficient (ns=not significant, *** $P<0.01$, **** $P<0.001$ ). Note the $\log _{10}$ axes
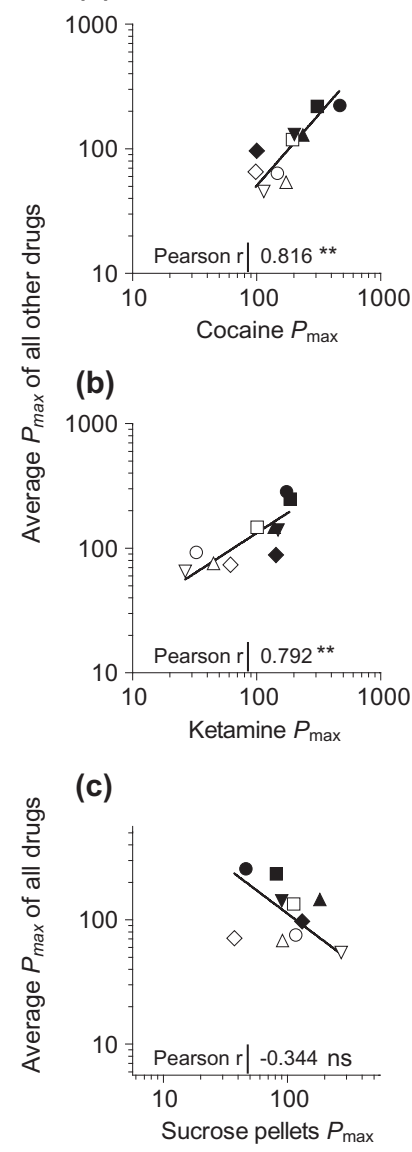

(d)

(g)

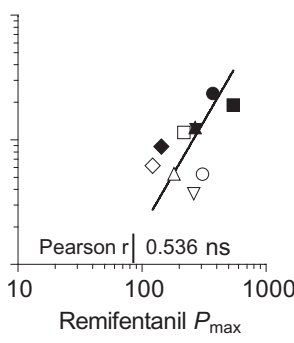

(e)

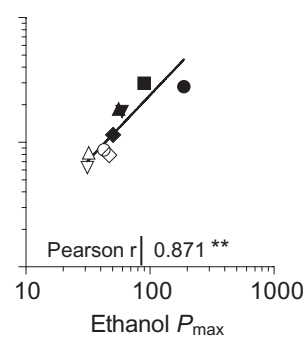

(f)

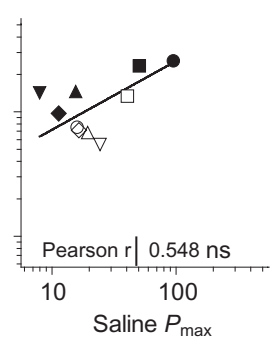

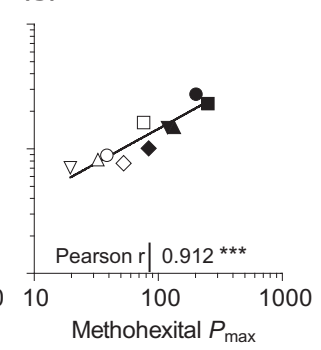

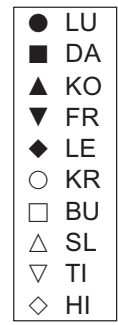

Table 4 Correlations between the $P_{\max }$ values or reinforcers earned at FR 10 for each drug and the average of the other drugs.

\begin{tabular}{|c|c|c|c|c|}
\hline & \multicolumn{2}{|l|}{$\mathrm{P}_{\max }$} & \multicolumn{2}{|c|}{ Reinforcers at FR 10} \\
\hline & Pearson's r & P value & Pearson's r & P value \\
\hline Cocaine & -0.816 & 0.004 & -0.030 & 0.935 \\
\hline Remifentanil & 0.536 & 0.110 & 0.294 & 0.410 \\
\hline Methohexital & 0.912 & $<0.001$ & 0.287 & 0.421 \\
\hline Ketamine & 0.792 & 0.006 & 0.184 & 0.611 \\
\hline Ethanol & 0.871 & 0.002 & -0.190 & 0.625 \\
\hline Saline & 0.548 & 0.101 & 0.360 & 0.308 \\
\hline Sucrose pellets & -0.344 & 0.331 & -0.295 & 0.408 \\
\hline
\end{tabular}

Correlations for cocaine, remifentanil, methohexital, ketamine and ethanol are between the value for that drug and the mean value of the other four drugs. Correlations for saline and sucrose pellets are between the value for saline or sucrose pellets, respectively, and the mean value for all five drugs.

tiveness of one drug were also likely to show relatively greater reinforcing effectiveness of the other drugs, whereas the monkeys that showed relatively lesser reinforcing effectiveness of one drug were likely to show relatively lesser reinforcing effectiveness of the other drugs.
The $P_{\max }$ value for sucrose pellets was negatively but not significantly correlated with the $P_{\max }$ for the other five drugs, suggesting, as shown earlier, that the demand for drug was unlikely to reflect a general tendency to respond more for reinforcers. The $P_{\max }$ values obtained with saline injections were positively correlated with the $P_{\max }$ values for the five drugs $(r=0.548)$, although this was not a significant correlation.

Similar calculations were used to determine whether these patterns would also be observed if reinforcers earned atFR 10 were used as a metric of drug self-administration. This was not the case. Individual subjects were unlikely to consistently earn more or fewer reinforcers than the average subject at an FR 10 (Fig. 2b). There were also no significant correlations between the number of reinforcers earned at an FR 10 for any of the drugs and these values for other drugs (Table 4), with all Pearson's $r$ values below 0.3 and none approaching statistical significance. Saline injections and sucrose pellets earned also did not correlate with reinforcers earned at an FR 10 for the five drugs (Table 4). Therefore, intake at the single, low price assessed at FR 10 did not offer the same resolving power to characterize the patterns described earlier as did demand functions, which measure behavior over a range of prices. 


\section{DISCUSSION}

In general, each of the five drugs served as reinforcers. Cocaine and remifentanil were ranked as the most effective reinforcers among this series of drugs; similarly, relatively inelastic demand for these two drugs has been shown in other studies using rhesus monkeys (e.g. WadeGaluska, Winger \& Woods 2007). Saline was the least effective reinforcing stimulus, and sucrose, methohexital, ketamine and ethanol fell between these two categories. Other studies have also shown methohexital, ketamine and ethanol to be relatively ineffective reinforcers compared with cocaine or remifentanil (Hursh \& Winger 1995; Winger, Galuska \& Hursh 2007). Food is sometimes reported to have greater reinforcing effectiveness than cocaine (e.g. Christensen et al. 2008a), while the reverse was true in this set of monkeys for cocaine and sucrose. This is likely due to the lack of a systematic food deprivation protocol in the current study (see methods section for description of the feeding protocol) as level of extra session feeding is known to affect food versus cocaine choice in monkeys (Nader \& Woolverton 1992; Negus 2003).

In addition to supporting earlier reports of differences among drugs with respect to their reinforcing effectiveness, the data also indicate that the monkeys differed in their sensitivities to the reinforcing effects of drugs. The monkeys that showed that one drug had relatively low reinforcing effectiveness tended to show that the other drugs also had relatively low reinforcing effectiveness. Said another way, some of the monkeys appeared to be very sensitive to the reinforcing effects of drugs, and the other monkeys appeared to be less sensitive to these effects. There was a continuum of general drug demand among this sample of 10 animals. Two monkeys (DA and LU) showed noticeably more inelastic demand for all five of the active drugs. This is a similar figure $(20 \%)$ to the rates of drug dependence found for many drugs in humans (Anthony et al. 1994) and for rates of cocaine addiction-like behavior in rats (Belin et al. 2008). These two monkeys also had the most inelastic demand for saline, suggesting that they either had a greater propensity to respond in general or that the light previously paired with the drug, the sound of the injection pump and sensations associated with an i.v. drug injection had greater conditional reinforcing effects for them. They were not systematically different from the other monkeys in their responding for sucrose pellets, indicating that their demand for the tested drugs was not based on a generally increased demand for all reinforcing stimuli.

The monkeys in this study had different histories of exposure to drugs. Nearly all of these histories consisted of the self-administration of moderate doses of drugs that were available during twice daily, two-hour sessions (see Table 1). Christensen et al. (2008b) showed that the reinforcing effectiveness of cocaine was enhanced with increased exposure to cocaine. Several other studies in rats have emphasized the importance of prolonged access to drugs of abuse before individual differences in addiction-like behaviors develop (Belin et al. 2008, 2009). In the current study, drug self-administration histories, in terms of the amount of drug taken or the variety of drugs taken, were not noticeably greater in the monkeys that showed greater demand for drugs. It is interesting to note, however, that the three monkeys that had experience with self-administration of the D3preferring dopamine agonists pramipexole and quinpirole, as well as with the D2 agonist sumanirole, were the monkeys that showed the most demand for the drugs used in the current study. The exposure to the dopamine agonists occurred in a study in which the reinforcing effects of these drugs were evaluated in comparison with cocaine. As can be determined from the table, LU and DA, the monkeys showing the most demand for drugs, selfadministered substantial doses of the D3-preferring agonists, whereas $\mathrm{KO}$, the monkey that ranked just below LU and DA in drug demand, did not demonstrate a reinforcing effect of the dopamine agonists (Koffarnus, Collins \& Woods, unpublished data). This is provocative information and among several issues that suggest future studies. Determining demand for drugs in animals that are initially drug-naïve and subsequently, evaluating drug demand throughout a series of specific drug exposures will provide needed information about the contribution that amount and duration of drug history make in the development of individual differences in sensitivity to the reinforcing effects of drugs. This can also answer the question of whether exposure to specific drugs appears to alter drug demand in some or all animals.

Anthony et al. (1994) surmised that the transition from drug use to drug dependence in humans could be impacted by the reinforcing effects of the drugs as well as by other factors including relative availability, opportunity to use, cost, social factors and individual vulnerabilities related to drug use. In the current study, the factors of availability, opportunity, cost and presumably, social factors were held constant, leaving reinforcing effects and individual vulnerability as the primary variables. Although no attempt was made here to measure drug dependence or drug addiction in these monkeys, and it is not clear how this could be accomplished, the data nevertheless strongly support the supposition of Anthony et al. (1994) that differences in sensitivity to reinforcing effects of the drugs may be a critical factor in the development of drug abuse in humans.

There is support in the human literature for these findings of a general rather than a specific responsiveness to 
drugs' reinforcing effects. Recent epidemiological data indicate that the primary drug of abuse at treatment entry varies dramatically among regions of the United States, suggesting that many drug users simply use whatever drugs are locally available (National Institute on Drug Abuse 2010). In an earlier study of male twin pairs, Tsuang et al. (1998) found that abuse of any particular drug was associated with a substantial increase in the likelihood of abusing every other type of drug. These investigators found that genetic and environmental factors both played a role in the shared vulnerability to drug abuse. This finding raises another potential use of the demand procedure-identifying specific phenotypes for genetic studies. Although increasingly sophisticated methods are being developed for evaluating genetic data, assessing drug-abusing phenotypes remains an issue that can require multiple determinations of drug use in substantial numbers of individuals (e.g. Johnson et al. 2008). Demand functions have been frequently obtained in humans (e.g. Greenwald 2008; MacKillop et al. 2009), and these procedures could be used to place numerical values (e.g. $P_{\max }$ values) on the sensitivity of randomly selected individuals to the reinforcing effects of drugs. Applying these sensitivity measures to genome-wide association data sets could dramatically sharpen the ability to apply genetic information to the presence of or likelihood of developing drug abuse.

An ongoing question has been whether use of some 'softer' drugs may lead individuals to eventual use of 'harder' drugs (e.g. Wagner \& Anthony 2002). Rather than serving as 'gateway drugs', use of these less reinforcing compounds may simply indicate enhanced responsiveness of an individual to the reinforcing effects of psychoactive drugs. Those who go on to develop addiction to cocaine or heroin may have greater sensitivity to the reinforcing effects of drugs in general than do people who stop their use with alcohol or marijuana.

The findings that a small sample of rhesus monkeys demonstrates a clear continuum of sensitivity to the reinforcing effects of drugs have several interesting implications about the basis of drug abuse, and assessing these reinforcing effects with demand functions may provide an effective technique for evaluating differential susceptibility to the reinforcing effects of drugs. Since these techniques can be employed in both animal and human populations, the cross-species relevance of findings can be evaluated in the near future.

\section{Acknowledgements}

The authors thank Susan Pouliot and Kathy Carey for their technical assistance and Steven Hursh for his assistance with ongoing $P_{\max }$ calculations. This work was supported by Public Health Service Grants to GW
(DA015449 and DA023992) and training grant support to MK (DA007267).

\section{DECLARATIONS OF INTEREST}

The authors declare that no financial support or compensation has been received from any private individual or corporation, and we have no personal financial holdings that constitute a real or perceived conflict of interest in this work.

\section{Authors Contribution}

MK and GW conceived and designed the study. AH and GW were responsible for acquisition of the data and overseeing the study in progress. AH assimilated the information included in Table 1. MK designed the data analysis procedures and carried out the data analysis. GW drafted the article, and MK and AH revised it. All authors reviewed the article and approved the final version for submission.

\section{References}

Anthony JC, Warner LA, Kessler RC (1994) Comparative epidemiology of dependence on tobacco, alcohol, controlled substances, and inhalants: basic findings from the National Comorbidity Survey. Exp Clin Psychopharmacol 2:244-268.

Belin D, Balado E, Piazza PV, Deroche-Gamonet V (2009) Pattern of intake and drug craving predict the development of cocaine addiction-like behavior in rats. Biol Psychiatry 65:863-868.

Belin D, Mar AC, Dalley JW, Robbins TW, Everitt BJ (2008) High Impulsivity predicts the switch to compulsive cocaine-taking. Science 320:1352-1355.

Christensen CJ, Silberberg A, Hursh SR, Huntsberry ME, Riley AL (2008a) Essential value of cocaine and food in rats: tests of the exponential model of demand. Psychopharmacology 198:221-229.

Christensen CJ, Silberberg A, Hursh SR, Roma PG, Riley AL (2008b) Demand for cocaine and food over time. Pharmacol Biochem Behav 91:209-216.

Flagel SB, Watson SJ, Akil H, Robinson TE (2008) Individual differences in the attribution of incentive salience to a rewardrelated cue: influence on cocaine sensitization. Behav Brain Res 186:48-56.

Greenwald MK (2008) Behavioral economic analysis of drug preference using multiple choice procedure data. Drug Alcohol Depend 93:103-110.

Hursh SR, Silberberg A (2008) Economic demand and essential value. Psychol Rev 115:186-198.

Hursh SR, Winger G (1995) Normalized demand for drugs and other reinforcers. J Exp Anal Behav 64:373-384.

Johnson C, Drgon T, Liu Q-R, Zhang P-W, Walther D, Li C-Y, Anthony JC, Ding Y, Eaton WW, Uhl GR (2008) Genome wide association for substance dependence: convergent results from epidemiological and research volunteer samples. BMC Med Genet 9:113.

MacKillop J, Murphy JG, Tidey JW, Kahler CW, Ray LA, Bickel WK (2009) Latent structure of facets of alcohol reinforcement from a behavioral economic demand curve. Psychopharmacology 203:33-40. 
Nader MA, Woolverton WL (1992) Choice between cocaine and food by rhesus monkeys: effects of conditions of food availability. Behav Pharmacol 3:635-638.

National Institute on Drug Abuse (2010) Epidemiologic Trends in Drug Abuse, Proceedings of the Community Epidemiology Work Group, Vol. 1. May, 2010, NIH Pub. No. 10-7421. Bethesda, MD: NIH, DHHS.

National Research Council (1996) Guide for the Care and Use of Laboratory Animals. Washington DC: National Academy Press.

Negus SS (2003) Rapid assessment of choice between cocaine and food in rhesus monkeys: effects of environmental manipulations and treatment with d-amphetamine and flupenthixol. Neuropsychopharmacology 28:919-931.

Osby U, Liljenberg J, Kokcum I, Gunnar A, Terenius L (2010) Genes and alcohol. Eur Psychiatry 25:281-283.

Piazza PV, Deminiere JM, Maccari S, Le Moal M, Simon H (1989) Factors that predict individual vulnerability of amphetamine self-administration. Science 245:1511-1513.

Piazza PV, Deminiere JM, Maccari S, Mormede P, Le Moal M, Simon H (1990) Individual reactivity to novelty predicts probability of amphetamine self-administration. Behav Pharmacol $1: 339-345$.
Stephens DN, Duka T, Crombag HS, Cunningham CL, Heilig M, Crabbe JC (2010) Reward sensitivity: issues of measurement, and achieving consilience between human and animal phenotypes. Addict Biol 15:145-168.

Tsuang MT, Lyons MJ, Meyer JM, Doyle T, Eisen SA, Goldberg J, True W, Lin N, Toomey R, Eaves L (1998) Co-occurrence of abuse of different drugs in men: the role of drug-specific and shared vulnerabilities. Arch Gen Psychiatry 55:967972.

Uhl GR (2006) Molecular genetics of addiction vulnerability. NeuroRx 3:295-301.

Wade-Galuska T, Winger G, Woods JH (2007) A behavioral economic analysis of cocaine and remifentanil selfadministration in rhesus monkeys. Psychopharmacology 194:563-572.

Wagner FA, Anthony JC (2002) Into the world of illegal drug use: exposure opportunity and other mechanisms linking the use of alcohol, tobacco, marijuana, and cocaine. Am J Epidemiol 155:918-925.

Winger G, Galuska CM, Hursh SR (2007) Modification of ethanol's reinforcing effectiveness in rhesus monkeys by cocaine, flunitrazepam, or gamma-hydroxybutyrate. Psychopharmacology 193:587-598. 